University of Georgia

Mathematics Preprint Series

No. 2, Volume 4 (1996)

To appear in Physica D

\title{
Bispectral Darboux Transformations: The Generalized Airy Case
}

\author{
Alex Kasman and Mitchell Rothstein \\ Department of Mathematics \\ University of Georgia \\ Athens, GA 30602
}

\begin{abstract}
This paper considers Darboux transformations of a bispectral operator which preserve its bispectrality. A sufficient condition for this to occur is given, and applied to the case of generalized Airy operators of arbitrary order $r>1$. As a result, the bispectrality of a large family of algebras of rank $r$ is demonstrated. An involution on these algebras is exhibited which exchanges the role of spatial and spectral parameters, generalizing Wilson's rank one bispectral involution. Spectral geometry and the relationship to the Sato grassmannian are discussed.
\end{abstract}

\section{Introduction}

An ordinary differential operator, $L\left(x, \partial_{x}\right)$, is said to be bispectral [4] if it has an eigenfunction $\psi(x, z)$ satisfying a pair of eigenvalue equations

$$
L(x, \partial) \psi(x, z)=f(z) \psi(x, z) \quad Q\left(z, \partial_{z}\right) \psi(x, z)=g(x) \psi(x, z)
$$

for non-constant functions $f$ and $g$. This property was investigated by Duistermaat and Grünbaum [3], who identified all bispectral Schrödinger operators: $L=\partial^{2}+V(x)$. In [15], Wilson considered the more general question of classifying bispectral commutative rings of ordinary differential operators. That is, he considered rings $\mathcal{L}$ and $\mathcal{Q}$ of operators in the variable $x$ and $z$ respectively such that there exists a function $\psi(x, z)$ for which Equation (11) holds for all $L \in \mathcal{L}$ and $Q \in \mathcal{Q}$. 
Following [2], one defines the rank of a commutative ring of ordinary differential operators to be the greatest common divisor of the orders of its elements. Wilson classified all maximal bispectral rings of rank one:

Theorem 1.1: [Wilson, 15]] Let $\mathcal{R}$ be a maximal rank one commutative algebra of ordinary differential operators. Then $\mathcal{R}$ is bispectral if and only if its spectral curve is a rational curve with no singularities other than cusps.

This result was proved, in part, through the introduction of an involution on a subset $G r^{a d}$ of the grassmannian $G r^{1}$ generally used in the investigation of the KP hierarchy 13]. By the work of Sato, Segal, Wilson and others [13] one knows how to associate to a point $W \in G r^{1}$ a commutative ring of operators and the Baker function $\psi_{W}(x, z)$ which is a common eigenfunction of the operators in the ring. Then $G r^{a d}$ is the subspace of $G r^{1}$ consisting of suitably normalized points whose corresponding ring is the affine coordinate ring of a rational curve with cusps, minus a point at $\infty$. Wilson showed that $G r^{a d}$ is equipped with an involution, $\beta: G r^{a d} \rightarrow G r^{a d}$, with the property that

$$
\psi_{\beta(W)}(x, z)=\psi_{W}(z, x)
$$

and consequently that the corresponding rings are bispectral. (Essentially, this is the involution which exchanges the rings $\mathcal{L}$ and $\mathcal{Q}$.)

The classification of bispectral algebras in higher rank appears to be more subtle [1, 6]. There is, however, a well-known method for constructing new commutative algebras of differential operators from a given one: Darboux transformation. (See, for example, [10, 17, 19] for previous results relating these transformations to the bispectral problem.) Under suitable hypotheses, described below, Darboux transformations preserve bispectrality. In this paper we will explore this method in the case of generalized Airy operators.

The general approach is the following. Let $\mathcal{D}$ denote the ring of ordinary differential operators with coefficients in $\mathbf{C}(x)$. Given a function $\psi(x, z)$, consider the equation

$$
T(x, \partial) \psi=T^{b}\left(z, \partial_{z}\right) \psi,
$$

for $T$ and $T^{b}$ in $\mathcal{D}$. Define

$$
\mathcal{A}_{\psi} \subset \mathcal{D}
$$

to be the set of operators $T$ for which there exists $T^{b}$ such that Equation (3) holds. Note that $\mathcal{A}_{\psi}$ is a subalgebra of $\mathcal{D}$. If $\psi$ is sufficiently general, in 
particular if $\psi$ is a bispectral eigenfunction satisfying equation (11), then the map

$$
T \mapsto T^{b}
$$

is an anti-isomorphism from $\mathcal{A}_{\psi}$ to $\mathcal{A}_{\tilde{\psi}}$, where

$$
\tilde{\psi}(x, z)=\psi(z, x) .
$$

Given a pair of operators $T$ and $L$, define an algebra of polynomials

$$
R_{T, L}:=\{p(z) \in \mathbf{C}[z] \mid T p(L) \subset \mathcal{D} T\} .
$$

Now assume Equation (四). For reasons explained in [15], it suffices to assume that $L$ and $Q$ are normalized, by which we mean that the top two coefficients are, respectively, constant and zero. It then follows that $L$ and $Q$ have coefficients in the field of rational functions $\mathbf{C}(x)$ (resp. $z$ ), and that $f(z)$ and $g(x)$ are polynomials. For $T \in \mathcal{A}_{\psi}$, let $a(x)$ (resp. $\left.a^{b}(z)\right)$ be the leading coefficient of $T\left(\operatorname{resp} . T^{\mathrm{b}}\left(z, \partial_{z}\right)\right)$ and

$$
\tilde{T}:=\frac{1}{a(x)} T \quad \tilde{T}^{b}:=\frac{1}{a^{b}(z)} T^{b}\left(z, \partial_{z}\right) .
$$

Then we immediately have the following proposition.

Proposition 1.2: Given $p_{1} \in R_{T, L}$ and $p_{2} \in R_{T^{b}, Q}$, let

$$
\begin{gathered}
P_{1}:=\tilde{T} p_{1}(L) \tilde{T}^{-1} \\
P_{2}:=\tilde{T}^{b} p_{2}(Q) \tilde{T}^{b}-1
\end{gathered}
$$

and let

$$
\phi(x, z):=\frac{1}{a(x) a^{b}(z)} T(\psi(x, z)) .
$$

Then $P_{1}$ and $P_{2}$ are normalized ordinary differential operators, satisfying

$$
\begin{gathered}
P_{1} \phi=p_{1}(f(z)) \phi \\
P_{2}\left(z, \partial_{z}\right) \phi=p_{2}(g(x)) \phi .
\end{gathered}
$$

Corollary 1.3: Given a bispectral triple $(L, Q, \psi)$ satisfying Equation 1 , then for $T \in \mathcal{A}_{\psi}$ such that the rings $R_{T, L}$ and $R_{T^{b}, Q}$ are non-trivial, the ring

$$
\mathcal{R}_{T, L}:=\left\{\tilde{T} p(L) \tilde{T}^{-1} \mid p \in R_{T, L}\right\}
$$

is an algebra of normalized bispectral operators, isomorphic to $R_{T, L}$. 
Thus one has a strategy for obtaining new bispectral algebras from a given bispectral operator, namely to find $T \in \mathcal{A}_{\psi}$ such that the rings $R_{T, L}$ and $R_{T^{\mathrm{b}}, Q}$ are non-trivial. Each such $T$ then gives a bispectral Darboux transformation of the bispectral operator $p(L)$ for $p \in R_{T, L}$.

For instance, Wilson's result, though not stated as such, is essentially the following. Taking $\psi(x, z)=e^{x z}, \mathcal{A}_{\psi}$ is the Weyl algebra of differential operators with polynomial coefficients. Indeed, in that case the map $T \mapsto$ $T^{b}$ is the standard antiautomorphism of the Weyl algebra exchanging $\partial$ and $x$.

Proposition 1.4: Let $\lambda_{1}, \ldots, \lambda_{n}$ be (not necessarily distinct) complex numbers and let $p_{1}(x), \ldots, p_{n}(x)$ be polynomials. Let $\phi_{i}=p_{i}(x) e^{\lambda_{i} x}$. Let $\bar{K}$ be the operator

$$
\bar{K}(u):=e^{-x \sum \lambda_{i}}\left|W r\left(\phi_{1}, \ldots, \phi_{n}, u\right)\right|,
$$

where $\left|W r\left(\phi_{1}, \ldots, \phi_{n}, \cdot\right)\right|$ denotes the Wronskian operator. (Clearly $\bar{K}$ has polynomial coefficients.) Then

1. The rings $\mathcal{R}_{\bar{K}, \partial}$ and $\mathcal{R}_{\bar{K}^{\mathrm{b}}, \partial}$ are both nontrivial.

2. Every maximal, normalized rank one bispectral algebra is obtained in this way.

The purpose of the present paper is to extend part of the proposition above to the higher rank case. What we are able to prove at the present time is that if we replace $\partial$ by a generalized Airy operator

$$
L_{0}:=\partial^{r}-a_{r-2} \partial^{r-2} \cdots-a_{1} \partial-x
$$

for $r>1$ and $a_{i} \in \mathbf{C}$, then the analogue of part one of the proposition continues to hold, at least when $\lambda_{1}, \ldots, \lambda_{n}$ are distinct and $p_{1}, \ldots, p_{n}$ are polynomials of degree one. This analogue is the following. The operator $L_{0}$ is clearly bispectral. Indeed, if we define

$$
\hat{f}(x, z):=f(x+z),
$$

then any $f \in \operatorname{ker}\left(L_{0}\right)$ satisfies the equations

$$
L_{0}(x, \partial) \hat{f}(x, z)=z \hat{f}(x, z) \quad L_{0}\left(z, \partial_{z}\right) \hat{f}(x, z)=x \hat{f}(x, z) .
$$

Note that for nonzero $f$ belonging to $\operatorname{ker}\left(L_{0}\right), \mathcal{A}_{\hat{f}}$ contains $\partial$, since $\partial(\hat{f})=$ $\partial_{z}(\hat{f})$, and it contains $x$ by equation (18). Thus $\mathcal{A}_{\hat{f}}$ is again the Weyl algebra and so $L_{0}$ determines an involution of the Weyl algebra. Explicitly, this involution is the unique anti-automorphism given by

$$
x^{b}=L_{0} \quad \partial^{b}=\partial .
$$


Throughout the remainder of the paper we will consider $L_{0}$ fixed and define the involution $b$ by Equation 19 .

The main result of the paper, to be developed and proved below is

Theorem 1.5: Let $f_{1}(x), \ldots, f_{r}(x)$ be a basis for the kernel of $L_{0}$. Define $\bar{K}$ to be the Wronskian operator of the rn functions

$$
\phi_{i, j}:=\left.p_{i}\left(\partial_{z}\right)\left(\hat{f}_{j}\right)\right|_{z=\lambda_{i}}
$$

For $\lambda_{1}, \ldots, \lambda_{n}$ distinct and $p_{1}, \ldots, p_{n}$ polynomials of degree one, the rings $\mathcal{R}_{\bar{K}, L_{0}}$ and $\mathcal{R}_{\bar{K}^{b}, L_{0}}$ are both nontrivial and therefore bispectral.

One of our purposes here is to draw attention to the rather interesting subset of the Weyl algebra consisting of those elements $T$ for which both $\mathcal{R}_{T, L_{0}}$ and $\mathcal{R}_{T^{b}, L_{0}}$ are nontrivial. We conjecture that it corresponds by the construction above to the set of homogeneous finite dimensional subspaces of the space of finitely supported distributions in the complex plane, as in Wilson's case.

By a finitely supported distribution we mean a finite linear combination of operators of the form $\delta_{\lambda} \circ \partial_{z}^{j}, \lambda \in \mathbf{C}$, where $\delta_{\lambda}$ is the $\delta$-function evaluating its argument at $z=\lambda$. We call such a distribution homogeneous if it is supported at one point, and we call a space of distributions homogeneous if it has a homogeneous basis.

Let $\mathcal{S}$ be the space of finitely supported distributions in the $z$-plane, and let $G r_{h}(\mathcal{S})$ denote the set of finite dimensional homogeneous subspaces of $\mathcal{S}$. $G r_{h}(\mathcal{S})$ appears to be a rather interesting space from an algebro-geometric point of view. Proposition 1.4 implies that Wilson's bispectral involution takes place on a certain quotient of $G r_{h}(\mathcal{S})$. Notice that if equation (1) holds, then $L$ itself belongs to $\mathcal{A}_{\psi}$. Moreover, if $T$ is replaced by $T q(L)$, where $q(z)$ is any nonzero polynomial, then the rings $R_{T, L}$ and $R_{T^{b}, L}$ remain unchanged, as do their script counterparts. Now it is not hard to show that if $C \in G r_{h}(\mathcal{S})$, and $\lambda$ is a complex number such that $\delta_{\lambda} \notin C$ then

$$
\bar{K}_{C \oplus\left\langle\delta_{\lambda}\right\rangle}=\bar{K}_{C(z-\lambda)} \circ(\partial-\lambda)
$$

where $\bar{K}$ now refers to the operator in Proposition 1.4. Thus if $C \in G r_{h}(\mathcal{S})$ is a space of distributions containing a delta function, we can use the action of $\mathbf{C}[z]$ on $G r_{h}(\mathcal{S})$ to replace point $C$ with a point $C^{\prime}$ that contains no $\delta$ functions and produces the same bispectral algebras. Let us call $C$ minimal if it contains no $\delta$ functions. Then every point $C$ has a minimal representative in the above sense, so we can think of the minimal points as either a quotient or a subset of $G r_{h}(\mathcal{S})$. Proposition 1.4 implies that the rank one bispectral involution may be regarded as an involution on 
the minimal points of $G r_{h}(\mathcal{S})$. Moreover, the involution takes place on finite dimensional pieces of $G r_{h}(\mathcal{S})$, cut out by placing an upper bound on both the order $\bar{K}$ and the degree of its leading coefficient in Proposition 1.4. We conjecture that precisely the same sort of phenomenon occurs in the generalized Airy case, though here we have only established this fact for elements of $G r_{h}(\mathcal{S})$ having a certain form. The natural approach to extending these higher rank involutions to all of $G r_{h}(\mathcal{S})$ seems to consist of first making a study of $G r_{h}(\mathcal{S})$ as an infinite dimensional algebraic variety and then applying a continuity argument. This we hope to do in a future work.

\subsection{Notation}

The notation ":=" will be used to indicate an initial definition of the object on the left hand side. Angular brackets $\left\langle c_{i}\right\rangle$ indicate the linear space spanned over $\mathbf{C}$ by the basis elements $c_{i}$. The determinant of a square matrix $M$ is denoted $|M|$. The Wronskian matrix $W r(\vec{v})$ of an $n$ vector $\vec{v}$ is the $n \times n$ matrix whose first row is $\vec{v}$ and so that each row is the derivative of the previous row. The symbols $\partial_{t}$ will be used to indicate $\frac{\partial}{\partial t}$ and $\partial$ is just $\partial_{x}$. An ordinary differential operator $L$ in the variable $x$ is a polynomial in the symbol $\partial$ with coefficients which are functions of the variable $x$. Often, a differential operator will be indicated by the notation $L\left(x, \partial_{x}\right)$ to indicate an operator in the variable $x$ and $L\left(z, \partial_{z}\right)$ to indicate the same operator following the change of variables $x \rightarrow z$.

\section{The Operators $\bar{K}$ and $\bar{K}^{b}$}

First we prove a general lemma which will be used at several points throughout the paper. Note that our distributions are assumed to act in the $z$ variable, so that if $\psi$ is a function of $x$ and $z, c(\psi)$ is a function of $x$.

Lemma 2.1: $\quad$ Let $L(x, \partial)$ be an rth order differential operator with coefficients analytic in a neighborhood $\mathcal{U} \subset \mathbf{C}$. Let $\mathcal{V} \subset \mathbf{C}$ be an open subset and let $\psi_{1}(x, z), \ldots, \psi_{r}(x, z)$ be functions analytic in $\mathcal{U} \times \mathcal{V}$, such that

1. $L\left(\psi_{i}\right)=z \psi_{i}$ for $i=1, \ldots r$.

2. For all $\lambda \in \mathcal{V}, \psi_{1}(x, \lambda), \ldots, \psi_{r}(x, \lambda)$ are linearly independent functions of $x$.

Let $\mathcal{O}(\mathcal{U})$ be the space of analytic functions on $\mathcal{U}$ and $\mathcal{S}(\mathcal{V}) \subset \mathcal{S}$ be the space of distributions with support in $\mathcal{V}$. Then the map

$$
\begin{aligned}
\mathcal{S}^{r}(\mathcal{V}) & \longrightarrow \mathcal{O}(\mathcal{U}) \\
\left(c_{1}, \ldots, c_{r}\right) & \mapsto \sum_{i} c_{i}\left(\psi_{i}\right)
\end{aligned}
$$


is injective.

Proof: Arguing by contradiction, assume $\sum_{i} c_{i}\left(\psi_{i}\right)=0$, with at least one $c_{i}$ non-zero. Let $\lambda_{1}, \ldots, \lambda_{n}$ be the points at which the $c$ 's are supported. For $j=1, \ldots, n$, let $\mu_{j}$ be the highest order derivative occurring among the $c$ 's at $\lambda_{j}$. Then let $m(z)$ be the polynomial

$$
m(z):=\left(z-\lambda_{1}\right)^{\mu_{1}} \prod_{i=2}^{n}\left(z-\lambda_{i}\right)^{\mu_{i}+1} .
$$

Let $c_{i}^{\prime}:=c_{i} \circ m(z)$. Then $c_{i}^{\prime}=\alpha_{i} \delta_{\lambda_{1}}$ with $\alpha_{i} \neq 0$ if and only if $c_{i}$ has order $\mu_{1}$ at $\lambda_{1}$. In particular, $c_{1}^{\prime}, \ldots, c_{r}^{\prime}$ are not all zero. However, we have

$$
\begin{aligned}
0 & =m(L)\left(\sum_{i} c_{i}\left(\psi_{i}\right)\right) \\
& =\sum_{i} c_{i} \circ m(L)\left(\psi_{i}\right) \\
& =\sum_{i} c_{i} \circ m(z)\left(\psi_{i}\right) \\
& =\sum_{i} \alpha_{i} \psi_{i}\left(x, \lambda_{1}\right)
\end{aligned}
$$

This implies that the $\alpha$ 's are zero, which is a contradiction.

Fix a basis $\left\{f_{j} \mid 1 \leq j \leq r\right\}$ for ker $L_{0}$. Since the $(r-1)^{s t}$ coefficient of $L_{0}$ is 0 , the Wronskian determinant, $\left|W r\left(f_{1}, \ldots, f_{r}\right)\right|$ is a non-zero constant. Assume the basis has been chosen so that

$$
\left|W r\left(f_{1}, \ldots, f_{r}\right)\right|=1
$$

Definition 2.2: Define $\mathbf{D} \subset G r_{h}(\mathcal{S})$ to be set of all finite dimensional subspaces $C \subset \mathcal{S}$ having a basis of the form

$$
\left\{c_{i}:=\delta_{\lambda_{i}} \circ\left(\partial_{z}+\gamma_{i}\right) \mid \lambda_{i} \text { distinct }\right\}
$$

Note: The elements of $\mathbf{D}$ should be thought of as identifying a singular rational spectral curve with simple cusps at the points $\lambda_{i}$ and a line bundle given by $\left(\gamma_{1}, \ldots, \gamma_{n}\right)$ in the generalized Jacobian of that curve.

Fix an element $C \in \mathbf{D}$. For convenience, we will order the basis for $C$, and define

$$
\begin{aligned}
\vec{\phi} & :=\left(c_{1}\left(\hat{f}_{1}\right), \ldots, c_{1}\left(\hat{f}_{r}\right), \ldots, c_{n}\left(\hat{f}_{1}\right), \ldots, c_{n}\left(\hat{f}_{r}\right)\right) \\
& :=\left(\phi_{1}(x), \ldots, \phi_{N}(x)\right)
\end{aligned}
$$


where $N:=r n$ and $\hat{f}(x, z)=f(x+z)$. As an immediate corollary of Lemma 2.1 we have

Proposition 2.3: $\quad$ The $N$ functions, $\phi_{s}$, are linearly independent.

The operator $\bar{K}$ in the introduction is defined only up to a constant, as it depends on a choice of basis. To be precise we will define

$$
\bar{K} u(x)=\frac{ \pm 1}{\prod_{i<j}\left(\lambda_{i}-\lambda_{j}\right)}|W r(\vec{\phi}, u)|,
$$

where the sign will be specified in Theorem 2.4 below.

It follows from Proposition 2.3 that $|W r(\vec{\phi})| \neq 0$, so that $\bar{K}$ is indeed a differential operator of order $N$. We may thus consider the ordinary differential operator $K$

$$
K u(x)=\frac{\left|W r\left(\phi_{1}, \ldots, \phi_{N}, u\right)\right|}{\left|W r\left(\phi_{1}, \ldots, \phi_{N}\right)\right|} .
$$

Note that this is the unique, monic operator of order $N$ having the space $\left\langle\phi_{s}\right\rangle$ as its kernel.

The main result of this section is

\section{Theorem 2.4:}

1. $\bar{K}$ has polynomial coefficients.

2. We can (and do) choose the sign of $\bar{K}$ so that the coefficient of $\partial^{N}$ is a monic polynomial of degree $n$.

3. $\bar{K}^{b}$ also has degree $N$.

Before proving this theorem, it is convenient to modify the definition of the Wronskian matrix to take account of the present situation. Define a sequence of operators

$$
P_{j}=\partial^{l} L_{0}^{k}, \text { where } j=r k+l, 0 \leq l<r .
$$

Then $P_{j}$ is monic of degree $j$. Given any vector of functions $\vec{v}=\left(v_{1}(x), \ldots, v_{m}(x)\right)$, define the modified Wronskian matrix

$$
\tilde{W} r\left(v_{1}, \ldots, v_{m}\right):=\left(\begin{array}{c}
\vec{v} \\
P_{1} \vec{v} \\
\vdots \\
P_{m-1} \vec{v}
\end{array}\right) .
$$

Since $\left\{P_{j}\right\}$ is related to $\left\{\partial^{j}\right\}$ by a unipotent transformation, we clearly have

$$
\left|\tilde{W} r\left(v_{1}, \ldots, v_{m}\right)\right|=\left|W r\left(v_{1}, \ldots, v_{m}\right)\right|
$$


Lemma 2.5: Given $c=\delta_{\lambda} \circ\left(a \partial_{z}+b\right) \in \mathcal{S}$,

$$
W r\left(c\left(\hat{f}_{1}\right), \ldots, c\left(\hat{f}_{r}\right)\right)=(b I+a B(x+\lambda)) \Omega(x+\lambda)
$$

where $\Omega(x):=W r\left(f_{1}, \ldots, f_{r}\right)$ and

$$
B(x)=\left(\begin{array}{cccccc}
0 & 1 & & & & \\
& 0 & 1 & & & \\
& & \ddots & \ddots & & \\
& & & & 1 & \\
& & & & 0 & 1 \\
x & a_{1} & a_{2} & \cdots & a_{r-2} & 0
\end{array}\right) .
$$

Proof: Let $v=(b, a, \underbrace{0, \ldots, 0}_{r-2})$. Then

$$
\left(c\left(\hat{f}_{1}\right), \ldots, c\left(\hat{f}_{r}\right)\right)=v \Omega(x+\lambda) .
$$

Now $\Omega^{\prime}(x)=B(x) \Omega(x)$. Moreover, for $j \leq r-2, v B(x)^{j}=(\underbrace{0, \ldots, 0}_{j}, b, a, 0, \ldots, 0)$.

Thus

$$
W r\left(c\left(\hat{f}_{1}\right), \ldots, c\left(\hat{f}_{r}\right)\right)=\left(\begin{array}{c}
v \\
v B(x+\lambda) \\
\vdots \\
v B(x+\lambda)^{r-1}
\end{array}\right) \Omega(x+\lambda)
$$

But $\left(\begin{array}{c}v \\ v B \\ \vdots \\ v B^{r-1}\end{array}\right)=b I+a B$, so the result follows.

Now we give the

Proof of Theorem 2.4: For $i=1, \ldots, n$ and $j=0, \ldots n-1$, let $A_{i j}$ be the $r \times r$ matrix

$$
A_{i j}:=\alpha_{i j} I+\lambda_{i}^{r} B\left(x+\lambda_{i}\right),
$$

where

$$
\alpha_{i j}:=\gamma_{i} \lambda_{i}^{j}+j \lambda_{i}^{j-1}
$$

Given any $f \in \operatorname{ker}\left(L_{0}\right)$,

$$
\begin{aligned}
\left(c(\hat{f}), P_{1}(c(\hat{f})), \ldots, P_{N}(c(\hat{f}))\right)= & \left(c(\hat{f}), \ldots, c\left(\widehat{f^{(r-1)}}\right)\right) \\
& c(z \hat{f}), \ldots, c\left(z f^{(r-1)}\right), \ldots, \\
& c\left(z^{n-1} \hat{f}\right), \ldots, c\left(z^{n-1} f^{(r-1)}\right), \\
& \left.c\left(z^{n} \hat{f}\right)\right) .
\end{aligned}
$$


It then follows from Lemma 2.5 that

$$
\bar{K} u=\frac{ \pm 1}{\prod_{i<j}\left(\lambda_{i}-\lambda_{j}\right)}\left|\begin{array}{cc}
A & \vec{U} \\
-\Lambda & P_{N}(u)
\end{array}\right|
$$

where

$$
\begin{gathered}
\vec{U}=\left(\begin{array}{c}
u \\
P_{1}(u) \\
\vdots \\
P_{N-1}(u)
\end{array}\right), \\
\Lambda:=(-\gamma_{0} \lambda_{0}^{n}-n \lambda_{0}^{n-1},-\lambda_{0}^{n}, \underbrace{0, \ldots, 0}_{r-2}, \ldots,-\gamma_{n-1} \lambda_{n-1}^{n}-n \lambda_{n-1}^{n-1},-\lambda_{n-1}^{n}, \underbrace{0, \ldots, 0}_{r-2})
\end{gathered}
$$

and

$$
A:=\left(\begin{array}{cccc}
A_{10} & A_{20} & \ldots & A_{n 0} \\
A_{11} & & & \vdots \\
\vdots & & & \vdots \\
A_{1, n-1} & \ldots & \ldots & A_{n, n-1}
\end{array}\right)
$$

It follows immediately that $\bar{K}$ has polynomial coefficients. Moreover, if we let $A^{\prime}$ be the $n \times n$ submatrix of $A$ in which $x$ occurs, then

$$
\left|A^{\prime}\right|=\left(\prod_{i<j}\left(\lambda_{i}-\lambda_{j}\right)\right)\left(\prod_{i}\left(x+\lambda_{i}\right)\right) .
$$

Letting $A^{\prime \prime}$ be the complementary $(r-1) n \times(r-1) n$ submatrix, we have

$$
\left|A^{\prime \prime}\right|=\left(\prod_{i<j}\left(\lambda_{i}-\lambda_{j}\right)\right)^{r-1}
$$

Thus

$$
|A|=\left(\prod_{i<j}\left(\lambda_{i}-\lambda_{j}\right)\right)^{r} x^{n}+O\left(x^{n-1}\right),
$$

which proves assertion 2 . To prove 3 , let $K_{i, j}$ be the coefficient of $P_{i r+j}(u)$ in the determinant Equation 40. Then

$$
\bar{K}^{b}=\sum x^{i} \partial^{j} K_{i, j}\left(L_{0}\right)
$$

Since $\operatorname{deg} K_{n, 0}=\operatorname{deg}|A|=n$ by Equation 46, we must show that

$$
\operatorname{deg}\left(K_{i, 0}\right) \leq n \quad \text { and }
$$




$$
\operatorname{deg}\left(K_{i, j}\right)<n \text { for } j>0 \text {. }
$$

Labeling the rows of $A$ as $A_{0}, \ldots A_{N-1}$,

$$
K_{i j}= \pm\left|\tilde{A}_{(m)}\right| \text {, }
$$

where $m=i r+j$ and $\tilde{A}_{(m)}$ is the matrix obtained from $A$ by replacing $A_{m}$ with $\Lambda$. Then the same argument that proves assertion 2 proves that $\operatorname{deg}\left(K_{i, 0}\right) \leq n$. It also proves that $\operatorname{deg}\left(K_{i, j}\right)<n$ if $0<j<r-1$. Indeed, in that case the submatrix of $A$ which gave us the coefficient of $x^{n}$ has now acquired a repeated row. But if $j=r-1$, then we have removed one of the rows in which $x$ occurred, so in that case the degree also drops.

\subsection{Examples}

It follows from the proof of Theorem 2.4 that one may easily compute $\bar{K}$ and $\bar{K}^{b}$. In the last column of determinant Equation 40, replace $P_{r j+k}(u)$ with $z^{j} \xi^{k}$. The determinant is then a polynomial in $x, z, \xi$. To get $\bar{K}$, we make the replacement $z^{j} \xi^{k} \mapsto \partial^{k} L_{0}^{j}$, and to get $\bar{K}^{b}$ we make the same replacement after first switching $x$ and $z$.

Case $n=1$ and $r=2$ : In this case, we have $c=\delta_{\lambda} \circ(\partial+\gamma)$ and $L_{0}=\partial^{2}-x$. The determinant is

$$
-\left|\begin{array}{ccc}
\gamma & 1 & 1 \\
\lambda+x & \gamma & \xi \\
1+\gamma \lambda & \lambda & z
\end{array}\right|=-\xi+\gamma+\gamma^{2} \lambda-\lambda^{2}-\lambda x-\left(\gamma^{2}-\lambda\right) z+x z
$$

This gives

$$
\begin{aligned}
\bar{K} & =\left(x+\lambda-\gamma^{2}\right) \partial^{2}-\partial+\left(\gamma^{2}-\lambda-x\right) x+\gamma+\gamma^{2} \lambda-\lambda^{2}-\lambda x \\
\bar{K}^{b} & =(x-\lambda) \partial^{2}-\partial+(\lambda-x) x+\gamma+\gamma^{2} \lambda-\lambda^{2}-\left(\gamma^{2}-\lambda\right) x
\end{aligned}
$$

The point, which we will generalize, is that $\bar{K}^{b}$ is obtained from $\bar{K}$ by the involutive map

$$
(\lambda, \gamma) \mapsto\left(\gamma^{2}-\lambda, \gamma\right)
$$

In other words, $\bar{K}_{C}^{b}=\bar{K}_{C^{\beta}}$ for some $C^{\beta}$.

Case $n=1$ and $r=3$ : When $r=3$, the vacuum has a parameter,

$$
L_{0}=\partial^{3}-a \partial-x .
$$

With $c$ as in the previous example, the relevant determinant is

$$
\left|\begin{array}{cccc}
\gamma & 1 & 0 & 1 \\
0 & \gamma & 1 & \xi \\
\lambda+x & a & \gamma & \xi^{2} \\
1+\gamma \lambda & \lambda & 0 & z
\end{array}\right|=\begin{aligned}
& \\
& \gamma \xi-\xi^{2}-a \gamma z+\gamma^{3} z+\lambda z+x z \\
& \gamma-\gamma^{3} \lambda-\lambda^{2}-\lambda x+
\end{aligned}
$$


This gives

$$
\begin{aligned}
\bar{K}= & \left(x-a \gamma+\gamma^{3}+\lambda\right) \partial^{3}-\partial^{2}+\left(\gamma+a^{2} \gamma-a \gamma^{3}-a \lambda-a x\right) \partial \\
& +a-\gamma^{2}+a \gamma \lambda-\gamma^{3} \lambda-\lambda^{2}+a \gamma x-\gamma^{3} x-2 \lambda x-x^{2} \\
\bar{K}^{b}= & (x-\lambda) \partial^{3}-\partial^{2}+(\gamma+a \lambda-a x) \partial+a-\gamma^{2}+a \gamma \lambda- \\
& \gamma^{3} \lambda-\lambda^{2}-a \gamma x+\gamma^{3} x+2 \lambda x-x^{2}
\end{aligned}
$$

These correspond under the involution

$$
(\lambda, \gamma) \mapsto\left(a \gamma-\gamma^{3}-\lambda, \gamma\right)
$$

\section{Bispectrality}

Our aim now is to prove that for $\bar{K}=\bar{K}_{C}$ as defined in Equation 28, the rings $R_{\bar{K}, L_{0}}$ and $R_{\bar{K}^{\mathrm{b}}, L_{0}}$ are nontrivial. First consider the stablizer of $C$ in the polynomial ring

$$
R_{C}:=\{p(z) \in \mathbf{C}[z] \mid c \circ p \in C \text { for all } c \in C\} .
$$

It follows from the formula

$$
\delta_{\lambda} \circ \partial_{z} \circ p(z)=p(\lambda) \circ \delta_{\lambda} \circ \partial_{z}+p^{\prime}(\lambda) \circ \delta_{\lambda}
$$

that

$$
R_{C}=\left\{p(z) \mid p^{\prime}\left(\lambda_{i}\right)=0,1 \leq i \leq n\right\}
$$

Lemma 3.1: $R_{\bar{K}, L_{0}}=R_{C}$.

Proof: It is a standard fact that the left ideal $\mathcal{D} K$ is precisely the set of operators which annihilate the kernel of $K$. In particular, $p(z)$ belongs to $R_{\bar{K}, L_{0}}$ if and only if $K p\left(L_{0}\right)(c(\hat{f}))=0$ for every $f \in \operatorname{ker}\left(L_{0}\right)$ and $c \in$ $C$. Since $K p\left(L_{0}\right)(c(\hat{f}))=\bar{K} c(p(z) \hat{f})$, this is the same has saying $C \circ$ $\left.p(z)\left(k \widehat{e r(L}_{0}\right)\right) \subset C\left(k e \widehat{e r\left(L_{0}\right)}\right)$. By Lemma 2.1, this occurs if and only if $C \circ p(z) \subset C$.

It now follows from (61) that $R_{\bar{K}, L_{0}} \neq \mathbf{C}$ and in fact contains elements of every sufficiently high degree. Consequently,

$$
\mathcal{R}_{\bar{K}, L_{0}}:=\left\{K p\left(L_{0}\right) K^{-1} \mid p \in R_{\bar{K}, L_{0}}\right\}
$$

is a commutative ring of ordinary differential operators of rank $r$. We remark that this ring is the iterated Darboux transformation $[10]$ of $\mathbf{C}\left[L_{0}\right]$ by the zero eigenfunctions $\phi_{s}$. 
In fact, it is well-known that for any point $C \in G r_{h}(\mathcal{S}), R_{C}$ is nontrivial. Thus, to prove that $R_{\bar{K}_{C}^{b}, L_{0}}$ is also non-trivial it suffices to find a space $C^{\beta} \in G r_{h}(\mathcal{S})$ such that $\bar{K}_{C}^{b}=\bar{K}_{C^{\beta}}$.

Definition 3.2: Let $C^{\beta} \subset \mathcal{S}$ be the subspace

$$
C^{\beta}:=\left\{c \in \mathcal{S} \mid c(\hat{f}) \in \operatorname{ker} \bar{K}^{b} \forall f \in \operatorname{ker} L_{0}\right\} .
$$

An important alternative definition of $C^{\beta}$ is given as follows.

Lemma 3.3: $C^{\beta}=\left\{c \in \mathcal{S} \mid c \circ \bar{K}\left(z, \partial_{z}\right)=0\right\}$.

Proof: Using the definition (3) of the involution $b, c$ belongs to $C^{\beta}$ if and only if

$$
\begin{aligned}
0 & =\bar{K}^{b} \circ c(\hat{f}) \\
& =c \circ \bar{K}^{b}(\hat{f}) \\
& =c \circ \bar{K}\left(z, \partial_{z}\right)(\hat{f}) .
\end{aligned}
$$

By Lemma 2.1, this occurs if and only if $c \circ \bar{K}\left(z, \partial_{z}\right)=0$.

Lemma 3.4: $\quad C^{\beta}$ is at most $n$-dimensional.

Proof: By Theorem 2.4 (3), $\bar{K}^{b}$ has degree $N=r n$. Thus, its kernel is at most $N$ dimensional. However, by Proposition 2.3, the vector space $\left\{c(\hat{f}) \mid c \in C^{\beta} f \in \operatorname{ker} L_{0}\right\}$ has dimension $r \cdot \operatorname{dim} C^{\beta}$. Since this is contained in the kernel of $\bar{K}^{b}$ by definition, $\operatorname{dim} C^{\beta} \leq n$.

Now we make a general observation about Wronskians, which will enable us to conclude that the dimension of $C^{\beta}$ is exactly $n$.

Theorem 3.5: Let $\vec{g}:=\left(g_{1}(z), \ldots, g_{m}(z)\right)$ be a vector of analytic functions of $z$. Let $w(z):=\left|W r\left(g_{1}(z), \ldots, g_{m}(z)\right)\right|$ and let $Q\left(z, \partial_{z}\right)$ be the differential operator whose application to an arbitrary function $u(z)$ is given by $Q u=$ $\left|W r\left(g_{1}(z), \ldots, g_{m}(z), u\right)\right|$. Given $\lambda \in \mathbf{C}$ such that $w(\lambda)=0$ and $w^{\prime}(\lambda) \neq 0$, there exists a unique distribution of the form $c=\delta_{\lambda} \circ(\partial+\gamma)$ such that $c \circ Q=0$. Moreover, if we set

$$
Q=w(z) \partial_{z}^{m}-w^{\prime}(z) \partial_{z}^{m-1}+v(z) \partial_{z}^{m-2}+O\left(\partial_{z}^{m-3}\right)
$$

then

$$
\gamma=\frac{v(\lambda)-w^{\prime \prime}(\lambda)}{w^{\prime}(\lambda)}
$$

Proof: We have

$$
A_{1}:=\left.(\partial \circ Q)(u)\right|_{z=\lambda}=\left|\begin{array}{cc}
H & \vec{u} \\
\vec{g}(m+1) & u^{(m+1)}(\lambda)
\end{array}\right|
$$


and

$$
A_{2}:=\left.Q(u)\right|_{z=\lambda}=\left|\begin{array}{cc}
H & \vec{u} \\
\vec{g}(m) & u^{(m)}(\lambda)
\end{array}\right|
$$

where $\vec{g}(j)$ is the $1 \times m$ vector

$$
\vec{g}(j):=\left.\frac{d^{j}}{d z^{j}}\left(g_{1}, \ldots, g_{m}\right)\right|_{z=\lambda}
$$

$H$ is the $m \times m$ matrix

$$
H:=\left(\begin{array}{c}
\vec{g}(0) \\
\vdots \\
\vec{g}(m-1)
\end{array}\right)
$$

and $\vec{u}$ is the $m \times 1$ vector

$$
\vec{u}:=\left(\begin{array}{c}
u(\lambda) \\
u^{\prime}(\lambda) \\
\vdots \\
u^{(m-1)}(\lambda)
\end{array}\right)
$$

Since $|H|=0$, the "bottom right" corner of each matrix above does not affect the determinant and

$$
A_{1}+\gamma A_{2}=\left|\begin{array}{cc}
H & \vec{u} \\
\vec{g}(m+1)+\gamma \vec{g}(m) & 0
\end{array}\right|
$$

This determinant is zero (for arbitrary $u$ ) if and only if $\vec{g}(m+1)+\gamma \vec{g}(m) \in V$ for

$$
V:=\langle\vec{g}(s) \mid 0 \leq s \leq m-1\rangle \subset \mathbf{C}^{m} .
$$

Furthermore, since $w^{\prime}(\lambda) \neq 0$, the vectors $\vec{g}(s)$ for $s=0, \ldots, m$ span all of $\mathbf{C}^{m}$. Consequently, there is a unique $\gamma$ such that

$$
\vec{g}(m+1)=-\gamma g(m)+\sum_{i=0}^{m-2} \alpha_{i} \vec{g}(i) .
$$

Such a $\gamma$ gives us our $c$. On the other hand, a direct calculation shows that the highest order terms of $c_{\lambda} \circ Q$ vanish only if $\gamma$ has the form (65).

In the rank one case, the bispectral involution was shown to exchange the roles of the $\tau$-function, which is zero when the $x$-orbit leaves the big cell in the grassmannian, and the polynomial $q(z)$ which is zero at the singular points of the spectral curve [1, 15]. This pair of polynomials will be seen 
to satisfy the same relationship and will also play an important role in the present paper.

Definition 3.6: Let $\tau(x)=\tau_{C}(x)$ be the coefficient of the $\partial^{N}$ in $\bar{K}$. In particular,

$$
\tau(x):=\frac{ \pm 1}{\left(\prod_{i<j}\left(\lambda_{i}-\lambda_{j}\right)\right)^{r}}|A|
$$

is a monic polynomial of degree $n$ where $A$ is as defined by (37) and (43). Let $q_{C}(z)=q(z):=\prod_{i=1}^{n}\left(z-\lambda_{i}\right)$.

Let $\mathbf{D}(n)$ be the subset of $\mathbf{D}$ consisting of subspaces of dimension $n$. We immediately have

Corollary 3.7: If $C \in \mathbf{D}(n)$ and $\tau$ has distinct roots, then $C^{\beta} \in \mathbf{D}(n)$. Moreover, $C^{\beta}$ has support at the roots of the polynomial $\tau$.

Now we can deduce the main results. For convenience, we denote $\bar{K}^{\beta}:=$ $\bar{K}_{C^{\beta}}, \tau^{\beta}(x)=\tau_{C^{\beta}}$ and denote by $\mathbf{D}_{0}$ the subset

$$
\mathbf{D}_{0}:=\left\{C \in \mathbf{D} \mid \tau_{C} \text { has distinct roots }\right\} .
$$

Theorem 3.8: Assume $C \in \mathbf{D}_{0}$. Then

1. $\tau^{\beta}=q$

2. $\bar{K}^{b}=\bar{K}^{\beta}$.

Consequently, one may conclude that $\beta$ is an involution on $\mathbf{D}_{0}$.

Proof: By definition, $\bar{K}^{b}$ belongs to the ideal of operators annihilating $c(\hat{f})$ for $f \in \operatorname{ker}\left(L_{0}\right)$ and $c \in C^{\beta}$. By Corollary 3.7 and Theorem 2.4, both $\bar{K}^{b}$ and $\bar{K}^{\beta}$ have degree $N$. Thus there exists a non-zero rational function $\mu \in \mathbf{C}(x)$ such that

$$
\bar{K}^{b}=\mu \bar{K}^{\beta} .
$$

Observe next that that the the coefficients of $\bar{K}^{b}$ have degree at most $n$. This follows from the definition of $b$ and the fact that $\bar{K}$ has order $N$. Moreover, $c \circ \bar{K}^{b}=0$ for all $c \in C$. It follows that the leading coefficient of $\bar{K}^{b}$ vanishes on the support of $C$. This coefficient is easily seen to be monic, whence

$$
\bar{K}^{b}=q(x) \partial^{N}+b(x) \partial^{N-1}+O\left(\partial^{N-2}\right) .
$$

for some polynomial $b(x)$ of degree at most $n$. We also have

$$
\bar{K}^{\beta}=\tau^{\beta}(x) \partial^{N}-\tau_{\beta}^{\prime}(x) \partial^{N-1}+O\left(\partial^{N-2}\right) .
$$

Thus both claims are proved once we show that $\mu=1$. 
Again since $c \circ \bar{K}^{b}=0$,

$$
b(\lambda)=-q^{\prime}(\lambda)
$$

for all $\lambda$ in the support of $C$. Thus, there exists a constant $\xi \in \mathbf{C}$, such that

$$
b(x)=-q^{\prime}(x)+\xi q(x) .
$$

Then

$$
\begin{aligned}
\mu \tau^{\beta} & =q \\
\mu \tau^{\beta^{\prime}} & =q^{\prime}-\xi q .
\end{aligned}
$$

Taking quotients and integrating, we find that there exists $\rho \in \mathbf{C}^{*}$ such that

$$
\tau^{\beta}(x)=\rho q(x) e^{-\xi x} .
$$

Since $\tau^{\beta}$ and $q$ are polynomials, $\rho=1$ and $\xi=0$.

For any $c \in C, c(\hat{f}) \in \operatorname{ker} \bar{K}$ by definition of $K$. Furthermore, since $\tau^{\beta}=q$, it has distinct roots implying that $C \in \mathbf{D}_{0}$. Therefore, by the results above, $\left(\bar{K}^{\beta}\right)^{b}=\left(\bar{K}^{b}\right)^{b}=\bar{K}$ and so $c(\hat{f}) \in \operatorname{ker}\left(\bar{K}^{\beta}\right)^{b}$. Thus $C \subset$ $\left(C^{\beta}\right)^{\beta}$. Since both spaces have dimension $n$, they are equal.

Theorem 3.9: For any $C \in \mathbf{D}$ and $\bar{K}\left(=\bar{K}_{C}\right)$, the ring $\mathcal{R}_{\bar{K}, L_{0}}$ is a bispectral ring of operators.

Proof: By Proposition 1.2 it is sufficient to show that $R_{\bar{K}, L_{0}}$ and $R_{\bar{K}^{b}, L_{0}}$ both contain non-constant polynomials. It follows from Lemma 3.1 that $R_{\bar{K}, L_{0}}$ is non-trivial. One can show that if $Q_{t}$ is a family of elements in the Weyl algebra depending continuously on a parameter $t, Q_{t}$ having a fixed order in both $\partial$ and $x$ for all $t$, then the property of $R_{Q_{t}, L_{0}}$ being nontrivial is preserved under limits with respect to $t$. Thus we reduce to the case that $\tau$ has distinct roots. In such a case, Theorem 3.8 shows that $\bar{K}^{b}=\bar{K}_{C^{\beta}}$ for $C^{\beta} \in \mathbf{D}_{0}$ and so, once again, Lemma 3.1 demonstrates that $R_{\bar{K}^{b}, L_{0}}$ is non-trivial.

Definition 3.10: For any $f \in \operatorname{ker} L_{0}$, let

$$
\mathbf{f}_{C}(x, z):=\frac{1}{q(z)} K \hat{f}(x, z) .
$$

The map $f \mapsto \mathbf{f}_{C}$ takes ker $L_{0}$ to an $r$-dimensional space of common eigenfunctions for the operators $Q_{p}=K p\left(L_{0}\right) K^{-1} \in \mathcal{R}_{\bar{K}, L_{0}}$, satisfying

$$
Q_{p} \mathbf{f}_{C}(x, z)=p(z) \mathbf{f}_{C}(x, z) .
$$


As with the rank one bispectral involution [15], the action of $\beta$ exchanges the roles of the spectral and spatial parameter in the eigenfunctions, as demonstrated by the following result.

Proposition 3.11: For any $f \in \operatorname{ker} L_{0}$ and any $C \in \mathbf{D}_{0}$ the eigenfunctions $\mathbf{f}_{C}$ and $\mathbf{f}_{C^{\beta}}$ are related by the formula

$$
\mathbf{f}_{C}(x, z)=\mathbf{f}_{C^{\beta}}(z, x) .
$$

Proof:

$$
\begin{aligned}
\mathbf{f}_{C}(x, z) & =\frac{1}{q(z)} K \hat{f}(x, z) \\
& =\frac{1}{\tau(x) q(z)} \bar{K} \hat{f}(x, z) \\
& =\frac{1}{\tau(x) q(z)} \bar{K}^{b}\left(z, \partial_{z}\right) \hat{f}(x, z) \\
& =\frac{1}{\tau(x) q(z)} q(z) K^{\beta}\left(z, \partial_{z}\right) \hat{f}(x, z) \\
& =\frac{1}{q^{\beta}(x)} K^{\beta}\left(z, \partial_{z}\right) \hat{f}(x, z) \\
& =\mathbf{f}_{C^{\beta}}(z, x) .
\end{aligned}
$$

\section{True Rank}

The ring of operators $\mathcal{R}_{\bar{K}, L_{0}}$ clearly has rank $r$. However, if this ring is contained in a larger commutative ring of lower rank, it is said to only have "fake" rank $r$ 9, 19]. For example, if some other operator of order relatively prime to $r$ commutes with the elements of $\mathcal{R}_{\bar{K}, L_{0}}$, then it actually has "true" rank one. Since the bispectral operators contained in rank one bispectral rings have already been identified by Wilson [15], it is useful to note that the ring $\mathcal{R}_{\bar{K}, L_{0}}$ is not contained in a commutative ring of rank smaller than $r$.

Definition 4.1: The true rank of an ordinary differential operator is the rank of its centralizer in the ring of all ordinary differential operators. The true rank of a commutative ring of operators is the true rank of any of its elements.

Lemma 4.2: The centralizer of $L_{0}$ in the ring of ordinary differential operators is the polynomial ring $\mathbf{C}\left[L_{0}\right]$. In particular, $L_{0}$ has true rank $r$. 
Proof: Since $L_{0}$ is contained in the Weyl algebra of ordinary differential operators having polynomial coefficients, its centralizer is as well. Since $b$ is an algebra antiautomorphism, it suffices to prove the result for $L_{0}{ }^{b}$. But $L_{0}^{b}=x$, and it is well-known that the centralizer of $x$ in the Weyl algebra is $\mathbf{C}[x]$.

Lemma 4.3: If $X=Y_{1} Y_{2}$ and $\hat{X}=Y_{2} Y_{1}$ then $X$ and $\hat{X}$ have the same true rank.

Proof: If $Q$ is an operator commuting with $X$, then

$$
\begin{aligned}
0 & =Y_{2}[Q, X] Y_{1} \\
& =Y_{2} Q Y_{1} Y_{2} Y_{1}-Y_{2} Y_{1} Y_{2} Q Y_{1} \\
& =\left[Y_{2} Q Y_{1}, \hat{X}\right] .
\end{aligned}
$$

Let $r$ be the true rank of $\hat{X}$. Then, by (87), we have $\operatorname{ord}\left(Y_{2} Q Y_{1}\right) \equiv 0 \bmod r$. But, $\operatorname{ord}\left(Y_{2} Q Y_{1}\right)=\operatorname{ord}\left(Y_{2} Y_{1}\right)+\operatorname{ord}(Q)$ and since $\operatorname{ord}\left(Y_{2} Y_{1}\right) \equiv 0 \bmod r$ we conclude that $\operatorname{ord}(Q) \equiv 0 \bmod r$. Therefore, the true rank of $\hat{X}$ divides the true rank of $X$. Then, by symmetry, the true ranks are equal.

Note: Lemma 4.3 generalizes a previous result [9] demonstrating that Darboux transformations preserve true rank in the case $r=2$.

Proposition 4.4: The ring $\mathcal{R}_{\bar{K}, L_{0}}$ of ordinary differential operators has true rank $r$.

Proof: The operator $q^{2}\left(L_{0}\right) \in \mathbf{C}\left[L_{0}\right]$ has true rank $r$, according to Lemma 4.2. Furthermore,

$$
q^{2}\left(L_{0}\right) c(\hat{f})=c\left(q^{2}(z) \hat{f}\right)=0 \quad \forall c \in C, f \in \operatorname{ker} L_{0} .
$$

Thus, in particular, $q^{2}\left(L_{0}\right)=Q K$ for some $Q \in \mathcal{D}$. Then the operator $Q_{q^{2}}=K q^{2}\left(L_{0}\right) K^{-1} \in \mathcal{R}_{\bar{K}, L_{0}}$ is given by $K Q$ and has the same true rank as $q^{2}\left(L_{0}\right)$.

\section{Discussion}

\subsection{Examples}

The following example demonstrates the construction of bispectral algebras of rank $r=2$ in the case $n=1$. For $r=2$, there is only one choice for a generalized Airy vacuum: $L_{0}=\partial^{2}-x$. Thus, we may choose the classical Airy functions $f_{1}(x)=A i(x)$ and $f_{2}(x)=B i(x)$ as a basis for ker $L_{0}$. Consider the one-dimensional space $C=\left\langle\delta_{0} \circ\left(\partial_{z}+\gamma\right)\right\rangle$. A polynomial $p(z)$ 
stabilizes $c$ if and only if $p^{\prime}(0)=0$. Consequently, $R_{C}=\mathbf{C}\left[z^{2}, z^{3}\right]$. From Equation (52) $\tau(x)=x-\gamma^{2}$ and

$$
K=\partial^{2}+\frac{1}{\gamma^{2}-x} \partial+\frac{x^{2}-\gamma-\gamma^{2} x}{\gamma^{2}-x} .
$$

Then from Definition 3.10

$$
\mathbf{f}_{C}(x, z)=\left(1-\frac{\gamma}{z\left(\gamma^{2}-x\right)}\right) \hat{f}(x, z)+\frac{1}{z\left(\gamma^{2}-x\right)} \hat{f}^{\prime}(x, z)
$$

and from Equation (62)

$$
\mathcal{R}_{\bar{K}, L_{0}}=\mathbf{C}\left[L_{4}, L_{6}\right]
$$

where

$$
\begin{aligned}
L_{4}:= & \partial^{4}+\frac{2\left(-2-\gamma^{4} x+2 \gamma^{2} x^{2}-x^{3}\right)}{\left(\gamma^{2}-x\right)^{2}} \partial^{2} \\
& +\frac{-2\left(-4+2 \gamma^{3}-\gamma^{6}-2 \gamma x+3 \gamma^{4} x-3 \gamma^{2} x^{2}+x^{3}\right)}{\left(-\gamma^{2}+x\right)^{3}} \partial \\
& +x^{2}-\frac{8}{\left(-\gamma^{2}+x\right)^{4}}-\frac{4 \gamma}{\left(-\gamma^{2}+x\right)^{3}}+\frac{2}{-\gamma^{2}+x}
\end{aligned}
$$

and

$$
\begin{aligned}
L_{6}:= & \partial^{6}+\frac{3\left(-2-\gamma^{4} x+2 \gamma^{2} x^{2}-x^{3}\right)}{\left(\gamma^{2}-x\right)^{2}} \partial^{4} \\
& +\left(-6+\frac{24}{\left(-\gamma^{2}+x\right)^{3}}+\frac{6 \gamma}{\left(-\gamma^{2}+x\right)^{2}}\right) \partial^{3} \\
& +\left(3 x^{2}-\frac{72}{\left(-\gamma^{2}+x\right)^{4}}-\frac{18 \gamma}{\left(-\gamma^{2}+x\right)^{3}}+\frac{6 \gamma^{2}}{\left(-\gamma^{2}+x\right)^{2}}+\frac{9}{-\gamma^{2}+x}\right) \partial^{2} \\
& +\left(6 x+\frac{144}{\left(-\gamma^{2}+x\right)^{5}}+\frac{36 \gamma}{\left(-\gamma^{2}+x\right)^{4}}-\frac{12 \gamma^{2}}{\left(-\gamma^{2}+x\right)^{3}}-\frac{3\left(3+2 \gamma^{3}\right)}{\left(-\gamma^{2}+x\right)^{2}}-\frac{6 \gamma}{-\gamma^{2}+x}\right) \partial \\
& -1-x^{3}-\frac{144}{\left(-\gamma^{2}+x\right)^{6}}-\frac{36 \gamma}{\left(-\gamma^{2}+x\right)^{5}}+\frac{12 \gamma^{2}}{\left(-\gamma^{2}+x\right)^{4}} \\
& +\frac{3\left(3+2 \gamma^{3}\right)}{\left(-\gamma^{2}+x\right)^{3}}+\frac{3 \gamma}{\left(-\gamma^{2}+x\right)^{2}}-\frac{3 \gamma^{2}}{-\gamma^{2}+x}
\end{aligned}
$$

From Equation (54) we see that $C^{\beta}=\left\langle\delta_{\gamma^{2}} \circ\left(\partial_{z}+\gamma\right)\right\rangle$ and so $\tau^{\beta}(x)=x$, $R_{C^{\beta}}=\mathbf{C}\left[\left(z-\gamma^{2}\right)^{2},\left(z-\gamma^{2}\right)^{3}\right]$ and

$$
K^{\beta}=\partial^{2}-\frac{1}{x} \partial-x+\frac{\gamma}{x}-\gamma^{2} .
$$


Most significantly, computing $\mathbf{f}_{C^{\beta}}(x, z):=\frac{1}{z-\gamma^{2}} K^{\beta} \hat{f}(x, z)$ it is easily determined that

$$
\mathbf{f}_{C^{\beta}}(x, z)=\mathbf{f}_{C}(z, x)
$$

and so the operators in $\mathcal{R}_{C^{\beta}}:=K \mathbf{C}\left[\left(L_{0}-\gamma^{2}\right)^{2},\left(L_{0}-\gamma^{2}\right)^{3}\right] K^{-1}$ demonstrate the bispectrality of $\mathcal{R}_{\bar{K}, L_{0}}$.

In the case that $\gamma=0, C$ and $C^{\beta}$ are the same and so this is a fixed point of the involution. This example is discussed by Grünbaum [5] as a solution of the KP hierarchy. For arbitrary $\gamma$, the time dependent KP solution corresponding to this ring is a non-vanishing rational solution 14 and is described in 8 .

\subsection{Sato Grassmannian and KP Flows}

The composition of the Wilson's involution on $G r^{1}$ with the KP flows was shown to be a non-isospectral symmetry of the KP hierarchy and a linearizing map of the Calogero-Moser Particle System [7]. The higher rank involution presented here may similarly have interesting dynamic properties. Therefore, the embedding of $G r_{h}(\mathcal{S})$ into the Sato grassmannian $G r^{r}$ used in constructions of rank $r$ KP solutions [19] may be of interest.

Essentially, the construction above is a special case of the "dual construction" 88 which associates a point in $G r^{r}$ to subspaces of $\mathcal{S}^{r}$ whose dimension is divisible by $r$. Let

$$
F(x):=\left(W r\left(f_{1}(x), \ldots, f_{r}(x)\right)\right)^{-1}
$$

and $\mathcal{F}_{j}(x)$ denote the $j^{\text {th }}$ column of this matrix. Define the composition of an $r$-vector $\vec{v}=\left(v_{1}(z), \ldots, v_{r}(z)\right)$ with an element $c \in \mathcal{S}$ to be the element $c \circ \vec{v}=\left(c \circ v_{1}, \ldots, c \circ v_{r}\right) \in \mathcal{S}^{r}$

$$
(c \circ \vec{v})(\vec{w}):=c(\vec{v} \cdot \vec{w})
$$

Then $\hat{C} \subset \mathcal{S}^{r}$ is the $N$ dimensional subspace

$$
\hat{C}:=\left\langle c \circ \mathcal{F}_{j}(z) \mid c \in C 1 \leq j \leq r\right\rangle \text {. }
$$

To the vector space $\hat{C}$, we associate the point 1 W $W r^{r}$

$$
W:=\frac{1}{q(z)} \overline{V_{\hat{C}}}
$$

\footnotetext{
${ }^{1}$ In the case that $q(z)$ has a root on the unit circle $S^{1}$, the point $W$ achieved in this way is not contained in $G r^{r}$ as formulated in [19] since its elements were taken to be $L^{2}\left(S^{1}\right)$. This subtlety need not concern us here, however.
} 
where $V_{\hat{C}}$ is the null space of $\hat{C}$ in $(\mathbf{C}[z])^{r}$ and the overline indicates Hilbert closure in $H^{r}=L^{2}\left(S^{1}\right)^{r}$.

Proposition 5.3: The vector Baker function $\psi_{W}(x, z)$ and the eigenfunctions functions $\mathbf{f}_{j}(x, z):=\frac{1}{q(z)} K \hat{f}_{j}(x, z)$ corresponding to $C$ are related by the formula

$$
\psi_{W}(x, z)=\left(\mathbf{f}_{1}(x, z), \ldots, \mathbf{f}_{r}(x, z)\right) \cdot F^{-1}(z) .
$$

Furthermore, the ring of ordinary differential operators associated to $W$ is the $\operatorname{ring} \mathcal{R}_{\bar{K}, L_{0}}$.

Then, if we denote by $W$ the point in $G r^{r}$ corresponding to $C$ and by $W^{\beta}$ the point corresponding to $C^{\beta}$, Proposition 3.11 implies

Corollary 5.4: The action of the involution $\beta$ on vector baker functions is given by the formula

$$
\psi_{W}(x, z)=\psi_{W^{\beta}}(z, x) \cdot F^{-1}(x) \cdot F(z) .
$$

\subsection{Bispectral Flows}

In light of the remarks of the preceding section, it follows from the results of 12] that composing the distributions in $C$ with the function $e^{t z^{k}}$ induces the $r k^{\text {th }}$ flow of the KP hierarchy. In particular, $\mathcal{L}=K(t) L_{0}^{1 / r} K^{-1}(t)$ satisfies the equation

$$
\frac{\partial}{\partial t} \mathcal{L}=\left[\left(\mathcal{L}^{r k}\right)_{+}, \mathcal{L}\right]
$$

For $C \in \mathbf{D}_{0}$ and $t$ sufficiently small, $C \circ e^{t z^{k}} \in \mathbf{D}_{0}$. Consequently, one may conclude that the KP flows whose indices are zero modulo $r$ are a local symmetry of these bispectral solutions. Furthermore, as in [7], the composition of the KP flows with the bispectral involution is a non-isospectral symmetry. Finally, it can be shown that the bispectral involution is a symplectic map linearizing the Hamiltonian system determining the motion of the poles of these KP solutions. (We intend to discuss these results further in an upcoming paper.)

\subsection{General Remarks}

Associated to any commutative ring of ordinary differential operators of rank $r$ is its geometric spectral data, which are a rank $r$ vector bundle over a complex projective curve [8, 11]. The geometric spectral data corresponding to rank one bispectral rings are "unicursal" rational curves with any line bundle [15]. That is, any rational curve having singularities in 
the form of cusps with any line bundle is the geometric spectral data of some bispectral ring of operators. The spectral curves associated to the bispectral rings constructed in this paper are still rational curves with singularities only in the form of cusps. However, the bispectral rings presented here do not correspond to arbitrary rank $r$ vector bundles, but rather only very special bundles. Indeed, for given $n$ and $r$, our construction depends on $n+r-2$ parameters, which is in general fewer than the number of moduli for a bundle of rank $r$ on a singular rational curve of genus $n$.

Previous work has indicated a relationship between bispectrality and polynomial $\tau$-functions $[1,18]$. The results above continue to support such a relationship (Definition 3.6). Furthermore, the bispectral involution involves not only an exchange of the variables $x$ and $z$, but of the roles of the polynomials $\tau(x)$ and $q(z)$. Since the roots of $\tau(x)$ indicate places that the orbit of the point $W \in G r^{r}$ leave the big cell, and the roots of $q(z)$ indicate the singular points of the spectral curve, the bispectral involution can be roughly seen as an exchange of spectral curve with the $x$-orbit.

The rings $\mathcal{R}$ constructed in this paper contain operators normalized so that they are monic and have zero second coefficient. A change in variable or conjugation by a non-constant function would change this normalization but preserve bispectrality 15].

It has been noted previously [6] that in the case of the known bispectral operators associated to the KP hierarchy, one is able to determine a corresponding operator in $z$ whose eigenvalue is any antiderivative of the $\tau$ function. The same can be shown to be true in the present case. In particular, for every $p \in R_{C^{\beta}}$, there is an operator $Q_{p}\left(z, \partial_{z}\right)$ for which $\mathbf{f}_{C}(x, z)$ is an eigenfunction with eigenvalue $p(x)$. However, since $q^{\beta}(z)=\tau(z)$, we have by Equation 61 that $R_{C^{\beta}}$ is exactly the ring of polynomials whose derivatives are divisible by $\tau$.

Finally, in light of Wilson's result, it is natural to conjecture that given $L_{0}$, one may associate a bispectral ring to any finite dimensional homogeneous subspace $C \subset \mathcal{S}$ by defining $\mathcal{R}_{\bar{K}, L_{0}}$ as above. In particular, we conjecture that if we define $C^{\beta}$ as in Definition 3.2 for any $C \in G r_{h}(\mathcal{S})$, there always exists a nonzero $\mu \in \mathbf{C}(x)$ such that

$$
\bar{K}^{b}=\mu(x) \bar{K}^{\beta}
$$

which would imply that

$$
R_{\bar{K}^{b}, L_{0}}=R_{\bar{K}^{\beta}, L_{0}}=R_{C^{\beta}} \neq \mathbf{C} .
$$

For instance, suppose $r=2, C=\left\langle\delta_{\lambda_{1}} \circ\left(\partial+\gamma_{1}\right), \delta_{\lambda_{2}} \circ\left(\partial+\gamma_{2}\right)\right\rangle, \lambda_{1} \neq \lambda_{2}$, but $C$ does not belong to $\mathbf{D}_{0}$. Then one may check directly that there 
are two possibilities for $C^{\beta}$. Letting $\hat{\lambda}$ be the unique (repeated) root of $\tau$, $C^{\beta}$ is spanned by two distributions supported at $\hat{\lambda}$, of orders either $(3,0)$ or $(2,1)$. In both cases, $\bar{K}^{\beta}$ has order 4 , as does $\bar{K}^{b}$. This implies that Equation (99) holds.

Note: After the completion of this work, a preprint [1] was made available which presents similar results from a Lie theoretic point of view.

Acknowledgements The authors are grateful for friendly and helpful correspondence with Emma Previato, Tasso Kaper, Robin John Chapman and Martin Reinders.

\section{References}

[1] B. Bakalov, E. Horozov and M. Yakimov, "Highest Weight Modules of $W_{1+\infty}$, Darboux transformations and the bispectral problem", q-alg/9601017

[2] J.L. Burchnall and T.W. Chaundy, "Commutative Ordinary Differential Operators" Proc. London Math. Soc., 211 (1923) pp. 420-440

J.L. Burchnall and T.W. Chaundy, "Commutative Ordinary Differential Operators" Proc. Royal Soc. A, 118 (1928) pp. 557-583

[3] J.J. Duistermaat and F.A. Grünbaum, "Differential Equations in the Spectral Parameter" Communications in Mathematical Physics 103 (1986) pp. $177-240$

[4] F.A. Grünbaum, "The Limited Angle Problem in Tomography and some Related Mathematical Problems" in Bifurcation theory, Mechanics and Physics, D. Reidel Publishing, (1983) pp. 317-329

[5] F.A. Grünbaum, "The Kadomtsev-Petviashvili Equation: An Alternative Approach to the 'Rank Two' Solutions of Krichever and Novikov" Physics Letters A 139 (1989) pp. 146-150

[6] F.A. Grünbaum, "Time-Band Limiting and the Bispectral Problem" Communications on Pure and Applied Mathematics 157 (1994) pp. 307-328

[7] A. Kasman, "Bispectral KP Solutions and Linearization of CalogeroMoser Particle Systems", Communications in Mathematical Physics 172 (1995) pp. 427-448

[8] A. Kasman, "Darboux Transformations from $n$-KdV to KP", in preparation

A. Kasman, "Rank $r$ KP Solutions with Singular Rational Spectral Curves", Ph.D. Thesis, Boston University (1995)

[9] G. Latham and E. Previato, "Higher Rank Darboux Transformations", MSRI Preprint 05229-91, to appear in Proc. NATO ARW Nonsingular Limits of Dispersive Waves, Plenum Publishing 
[10] V.B. Matveev, "Darboux transformation and explicit solutions of the Kadomtcev-Petviaschvily Equation, Depending on Functional Parameters" Letters in Mathematical Physics 3 (1979), pp. 213-216

[11] E. Previato, "Seventy Years of Spectral Curves: 1923-1993" to appear in Proceedings of CIME 1993 Springer-Verlag, Lecture Notes in Physics

[12] E. Previato and G. Wilson, "Vector Bundles Over Curves and Solutions of the KP Equations" Proc. Sympos. Pure Math., 49 (1989), pp. 553-569

[13] G. Segal and G. Wilson, "Loop Groups and Equations of KdV Type" Publications Mathematiques No. 61 de l'Institut des Hautes Etudes Scientifiques (1985) pp. 5-65

[14] A.P. Veselov, "Rational Solutions of the KP Equation and Hamiltonian Systems" Communications of the Moscow Mathematical Society, Russian Math Surveys 35:1 (1980) pp. 239-240

[15] G. Wilson, "Bispectral Commutative Ordinary Differential Operators", J. reine angew. Math. 442 (1993) pp. 177-204

[16] P. Wright, "Darboux transformations, algebraic subvarieties of Grassmann manifolds, commuting flows and bispectrality", Ph.D. thesis, Berkeley 1987

[17] J. Zubelli, "Differential Equations in the Spectral Parameter for Matrix Differential Operators" Physica D 43 (1990) pp. 269-287

[18] J. Zubelli, "On the Polynomial Tau Function for the KP Hierarchy and the Bispectral Property" Letters in Mathematical Physics 24 (1992) pp. $41-48$

[19] J. Zubelli and F. Magri, "Differential Equations in the Spectral Parameter, Darboux Transformations and a Hierarchy of Master Symmetries for KdV", Communications in Mathematical Physics 141 (1991) pp. 329-351 